\title{
Low-Level Laser and Antimicrobial Photodynamic Therapy Reduce Peri-implantitis-related Microorganisms Grown In Vitro
}

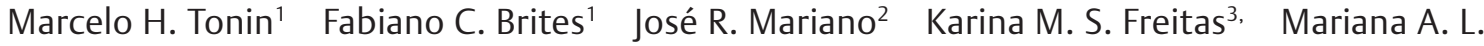 \\ Ortiz $^{4}$ Samira Salmeron ${ }^{5}$
}

1Department of Implantology, Ingá University Center, Maringá, Paraná, Brazil

2Department of Implantology, Unieuro University Center, Brasília, Brazil

${ }^{3}$ Department of Orthodontics, Ingá University Center, Maringá,

Paraná, Brazil

${ }^{4}$ Department of Microbiology, Ingá University Center, Maringá,

Paraná, Brazil

${ }^{5}$ Department of Periodontics and Implant Dentistry, Ingá University Center, Maringá, Brazil

Eur J Dent 2022;16:161-166.

\begin{abstract}
Address for correspondence Samira Salmeron, DDS, MSc, PhD, Department of Periodontics and Implant Dentistry, Ingá University Center, Uningá, Rod PR 317, 6114, 87035-510 Maringá, Paraná, Brazil (e-mail: salmeronsamira@gmail.com).
\end{abstract}

\section{Abstract}

Keywords

- decontamination

- lasers

- photodynamic therapy

- biofilm

- Staphylococcus aureus
Objective Currently, dental implants are a predictable treatment option for oral rehabilitation; however, complications such as peri-implant diseases are increasing every day. Thus, the aim of this study was to verify the efficacy, in vitro, of two protocols against cultures of periodontal biofilm and Staphylococcus aureus.

Material and Methods Petri dishes for each of the following groups were used: control groups (C)-plates inoculated with periodontal biofilm (C.B; $n=4)$ or S. aureus $(C . S A ; n=4)$ without any treatment; laser groups-plates inoculated with periodontal biofilm (low-level laser therapy [LLLT].B; $n=4$ ) or S. aureus (LLLT.SA; $n=4$ ) and treated with LLLT (660 nm, $30 \mathrm{~mW}, 50 \mathrm{~J} / \mathrm{cm}^{2}$, and 47 seconds); antimicrobial photodynamic therapy groups (aPDT)-plates inoculated with periodontal biofilm (aPDT.B; $n=4$ ) or S. aureus (aPDT.SA; $n=4$ ) and treated with aPDT (red laser $660 \mathrm{~nm}, 30 \mathrm{~mW}$, $50 \mathrm{~J} / \mathrm{cm}^{2}, 47$ seconds + toluidine blue O (TBO) $100 \mu \mathrm{g} / \mathrm{mL}$, and 1 minute). After treatments were performed, the contents of all plates were diluted and seeded for counting colony-forming units (CFUs).

Statistical Analysis Results were analyzed with one-way analysis of variance (ANOVA), Tukey's test, comparison of percentages, and independent $t$-tests with a $5 \%$ significance level.

Results Both treatments, LLLT and aPDT, significantly reduced the number of CFUs for the two types of culture, LLLT.B $\left(3.69 \times 10^{6} \pm 0.20\right)$, aPDT.B $\left(2.79 \times 10^{6} \pm 0.13\right)$, LLLT. SA $\left(4.10 \times 10^{6} \pm 0.12\right)$, and aPDT.SA $\left(3.23 \times 10^{6} \pm 0.10\right)$ when compared with control groups C.B $\left(5.18 \times 10^{6} \pm 0.43\right)$ and C.SA $\left(5.81 \times 10^{6} \pm 0.16 ; p=0.000\right)$. When treatment groups were compared separately, there was also a statistically significant difference $(p=0.000)$. None of the protocols were able to eliminate cultured microorganisms.

Conclusion The LLLT and aPDT protocols effectively reduced cultures of periodontal biofilm and S. aureus in vitro, with the superiority of aPDT. published online

October 1, 2021
DOI https://doi.org/ $10.1055 / \mathrm{s}-0041-1731926$ ISSN $1305-7456$ (c) 2021. The Author(s).

This is an open access article published by Thieme under the terms of the Creative Commons Attribution License, permitting unrestricted use, distribution, and reproduction so long as the original work is properly cited. (https:// creativecommons. org/licenses/by/4.0/).

Thieme Medical and Scientific Publishers Pvt. Ltd. A-12, 2nd Floor, Sector 2, Noida-201301 UP, India 


\section{Introduction}

Dental implants are currently a highly predictable treatment option for cases of oral rehabilitation, with high success rates $^{1}$ and survival. ${ }^{2}$ However, with the increase in the number of implants installed and the "aging" of these implants in the oral cavity, the number of complications also increases, for example, the peri-implant diseases. ${ }^{3}$

Peri-implant diseases, according to the recent classification, include peri-implant mucositis and peri-implantitis. ${ }^{4}$ Peri-implant mucositis is defined as an inflammatory lesion that affects the mucosa around the implants without loss of supporting bone. ${ }^{5}$ It is a reversible condition, once the biofilm is eliminated, characterized by bleeding on probing and visual signs of inflammation. ${ }^{5}$ Peri-implantitis presents a much more critical clinical situation. It is a pathological condition associated with a biofilm that involves the tissues around the implants, characterized by inflammation of the peri-implant mucosa, and subsequent and progressive bone loss. ${ }^{6}$

Recent evidence indicates that peri-implant mucositis is a precursor of peri-implantitis in the same way that gingivitis is of the periodontitis. ${ }^{7}$ Just as the biofilm is also considered the main etiological factor for peri-implant and periodontal diseases. ${ }^{4,7.8}$ Studies show that the peri-implantitis microbiota is similar to the periodontitis, ${ }^{9-11}$ including species of the red complex such as Porphyromonas gingivalis, Tannerella forsythia, and Treponema denticola. ${ }^{12}$ However, there may be differences in its composition ${ }^{10,13}$ with the identification of microorganisms not commonly found in periodontitis like Staphylococcus aureus, Staphylococcus epidermidis, and Candida spp..$^{14}$

The peri-implantitis microbiota is quite complex and heterogeneous, and its characterization remains incomplete. ${ }^{6}$ Its microbiome is characterized by a great diversity of microorganisms composed of aerobic gram-positive, anaerobic gram-negative, and fusiform pathogens. ${ }^{15}$ Persson and Renvert $^{10}$ identified a different bacterial profile in peri-implantitis, including P.gingivalis, S. aureus,Staphylococcus anaerobius, Streptococcus intermedius, Streptococcus mitis, T. forsythia, and Treponema socranskii. Sahrmann et al, ${ }^{15}$ in a systematic review and meta-analysis, found a high prevalence of Aggregatibacter actinomycetemcomitans and Prevotella intermedia in biofilms of implants with peri-implantitis compared with healthy implants. Rates of microorganisms identified in peri-implantitis that are less common in periodontitis, such as $S$. aureus and S. epidermidis, are reported in the literature. ${ }^{10}$ Infection by $S$. aureus, in particular, may be important in the development of peri-implantitis,,$^{10,16,17}$ since, during the formation of the biofilm, this microorganism acts as a primary colonizer, creating favorable conditions for late bacteria adherence and colonization. ${ }^{10}$

Thus, biofilm removal becomes essential in cases of peri-implantitis ${ }^{17,18}$; the prevention and treatment of this condition are even critical for the long-term stability of implants. ${ }^{18}$ It is suggested that the successful treatment of peri-implantitis is based on biofilm removal associated with the implant surface decontamination. In this context, several protocols for peri-implantitis treatment and implant surface decontamination are seen in literature. ${ }^{16}$ Chemical and mechanical methods have been proposed, but until now, no protocol is considered ideal for peri-implantitis treatment. ${ }^{19}$

The use of lasers and antimicrobial photodynamic therapy (aPDT) for decontamination of the titanium implant surfaces has been studied with very promising results. ${ }^{19,20}$ The great advantage of laser therapies is the absence of consequences seen in other methods such as local irritation and the development of bacterial resistance. ${ }^{16}$ However, the effect of the laser alone as a decontaminant of implant surfaces is controversial topic in literature. Some studies report good results $^{19,21}$ and others report no differences between laser and conventional treatments. ${ }^{22-24}$

In the last decades, the association of low-level lasers with photosensitizers has also been used to reduce or eliminate bacteria, known as aPDT. However, the large number of questions about the topic and the wide range of protocols described in the literature still do not assure the use of aPDT as an adjunct therapy in the treatment of peri-implantitis. Therefore, the present study aimed to verify the in vitro efficacy of two protocols, using low-level laser therapy (LLLT) and aPDT, against cultures of periodontal biofilm and S. aureus.

\section{Material and Methods}

\section{Microorganisms and Growth Conditions}

The sample of periodontal biofilm was frozen and obtained from the sample of the Laboratory of Microbiology at Ingá University Center, Uningá. ${ }^{20}$ It is a subgingival biofilm collected from a patient diagnosed with periodontitis. The $S$. aureus used was a standard strain (ATCC 25923) from the bacteria collection from the same laboratory at Ingá University Center, Uningá, which was frozen in Brain Heart Infusion (BHI) broth (Kasvi, São José dos Pinhais, Brazil) with 15\% glycerol. For use, the strain was thawed and transferred to Petri dishes containing culture medium and then incubated at $37^{\circ} \mathrm{C}$ for 24 hours for reactivation.

For the preparation of the inoculum, both the periodontal biofilm and the S. aureus strain were standardized by transferring colonies of these microorganisms to a tube with saline until turbidity corresponding to the 0.5 MacFarland scale, which corresponds to approximately $1.5 \times 10^{8}$ colony-forming units (CFU)/mL.

\section{Distribution of Experimental Groups}

Petri dishes containing $5 \mathrm{~mL}$ of standardized inoculums were distributed into the following groups, according to the culture of microorganisms: periodontal biofilm (B) or S. aureus (SA):

- Control groups: plates inoculated with periodontal biofilm (C.B; $n=4)$ or S. aureus (C.SA; $n=4$ ) without any treatment.

- Low-level laser therapy groups: plates inoculated with periodontal biofilm (LLLT.B; $n=4$ ) or S. aureus (LLLT.SA; $n=4$ ) and treated with low-level laser therapy.

- Antimicrobial photodynamic therapy groups: plates inoculated with periodontal biofilm (aPDT.B; $n=4$ ) or S. aureus (aPDT.SA; $n=4)$ and treated with aPDT. 
Standard microbiological tests were also performed to ensure the sterility of the culture medium.

\section{Photosensitizer Solution and Light Source}

The dye used was toluidine blue O (TBO; Sigma-Aldrich Brazil, São Paulo, Brazil), at a concentration of $100 \mu \mathrm{g} / \mathrm{mL}$, diluted in distilled water. The solution was prepared and handled under restricted light conditions. The laser used was the LLLT diode, InGaAlP (Whitening Lase II, DMC Equipment Ltda, São Carlos, Brazil). The irradiation parameters are described in $\boldsymbol{- T a b l e} \mathbf{1}$.

\section{Microorganism Culture Treatments}

\section{Low-level Laser Therapy}

The standardized inoculums of $5 \mathrm{~mL}$ were transferred to sterile Petri dishes according to the groups LLLT.B and LLLT. SA. The plates were irradiated with laser for 47 seconds, in scan mode, by a single operator, with tip/plate distance of $1 \mathrm{~cm}$, and according to the parameters described in $\boldsymbol{- T a b l e ~} \mathbf{1}$, inside the laminar flow chamber (-Fig. 1).

\section{Antimicrobial Photodynamic Therapy}

The standardized inoculums of $5 \mathrm{~mL}$ were transferred to sterile Petri dishes according to the groups aPDT.B and aPDT.SA. Then, $5 \mathrm{~mL}$ of the TBO solution was placed on the plates. After 1 minute, laser irradiation was performed for 47 seconds, in scan mode, by a single operator, with tip/plate distance of $1 \mathrm{~cm}$, according to the parameters described in $\boldsymbol{- T a b l e ~} 1$ (-Fig. 2).

\section{Colony-Forming Units Counting}

After treatments, the material from all plates, including the control groups, was diluted to a concentration of $10^{-3}$. Then, $100 \mu \mathrm{L}$ of these dilutions were transferred to blood agar plates, in duplicate, stored in a $\mathrm{CO}_{2} \mathrm{PVC}$ - polyvinyl chloride jar (Permution, Curitiba, Brazil) to guarantee the microaerophilic condition (candle jar technique, $5 \% \mathrm{CO}_{2}$ ), and placed in an incubator for 48 hours at $37^{\circ} \mathrm{C}$, allowing colonies to grow. After this period, CFUs were counted with the naked eye, by an experienced and calibrated examiner.

\section{Statistical Analysis}

The normality of the data was assessed by the Shapiro-Wilk test. The one-way analysis of variance (ANOVA) and post hoc Tukey's tests were used to compare the CFUs among the experimental groups. For the comparison of CFUs between treatments separately, the independent $t$-test was used. And for the comparison of CFUs reduction between test groups was performed the comparison of percentages. These tests were performed with Statistica software (Statistica for Windows version 10.0, Statsoft, Tulsa, Oklahoma, United States). The data were considered significant for $p<0.05$.

\section{Results}

There was a statistically significant difference in the number of CFUs among the groups evaluated, both for the periodontal biofilm and S. aureus. The treatments, LLLT and aPDT,
Table 1 Laser parameters

\begin{tabular}{|l|l|}
\hline Type of laser & Diode-InGaAlP \\
\hline Emission mode & Scan \\
\hline Wavelength $(\mathrm{nm})$ & 660 \\
\hline Power $(\mathrm{mW})$ & 30 \\
\hline Time $(\mathrm{s})$ & 47 \\
\hline Energy density $\left(\mathrm{J} / \mathrm{cm}^{2}\right)$ & 50 \\
\hline Tip area $\left(\mathrm{cm}^{2}\right)$ & 0.0028 \\
\hline Energy $(\mathrm{J})$ & 0.14 \\
\hline
\end{tabular}

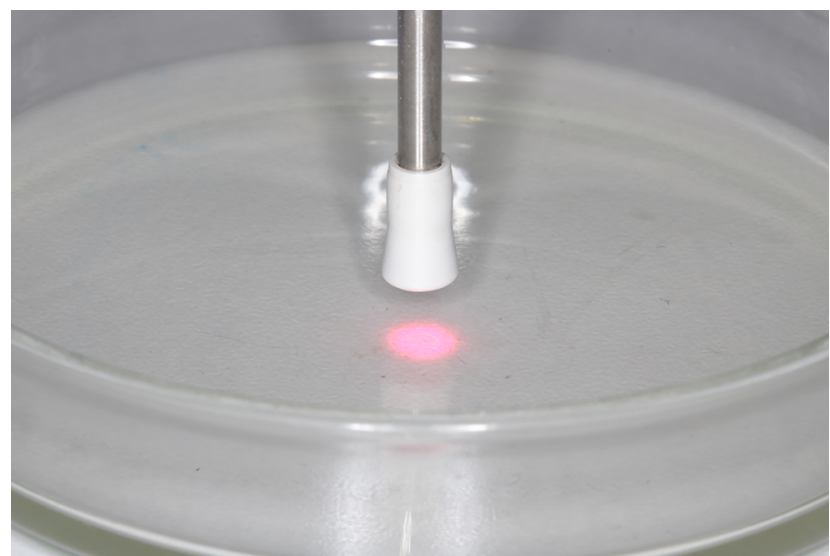

Fig. 1 Petri dish containing the microorganisms and being irradiated with low-level laser (red) according to the protocol.

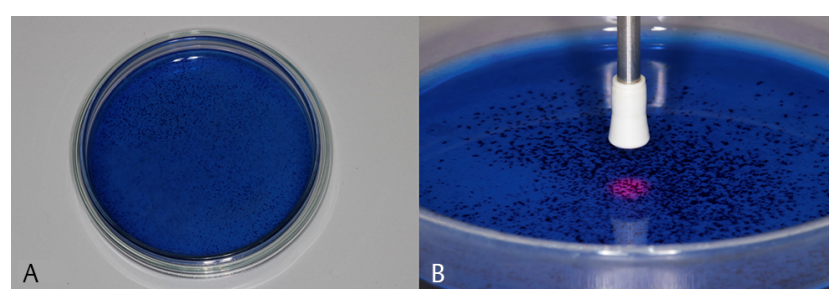

Fig. 2 (A) Preirradiation period. Petri dish containing the microorganisms and TBO applied for 1 minute before irradiation. (B) Irradiation. Petri dish containing the microorganisms immerged in TBO and being irradiated with low-level laser (red) according to the protocol. TBO, toluidine blue $\mathrm{O}$.

significantly reduced the number of CFUs of both cultures compared with controls $(p=0.000$; one-way ANOVA and post hoc Tukey's test; - Fig. 3). The antimicrobial action of both treatments was similar against periodontal biofilm and S. aureus with reduction of $28.77 \%$ and $29.44 \%$ CFUs, respectively, using LLLT treatment $(p=0.488$; comparison of percentages) and reduction of $46.14 \%$ CFUs of periodontal biofilm and $44.41 \%$ CFUs of $S$. aureus, using aPDT treatment ( $p=0.472$; comparison of percentages; - Table 2).

When treatments were compared separately, there was statistically significant difference ( $p=0.000$; independent $t$-test). A lesser number of CFUs for periodontal biofilm and S. aureus were observed after treatment with aPDT than with LLLT ( - Table 3). However, none of the treatments eliminated the microorganisms. 
Table 2 Values of reductions (P\%) in the number of CFUs considering control groups representing 100\% of microorganisms' growth

\begin{tabular}{|l|l|l|l|}
\hline Groups & CFUs & Reduction $(\mathrm{P} \%)$ & \multirow{2}{*}{$\boldsymbol{p}^{*}$} \\
\cline { 1 - 3 } LLLT-B & $71.23 \%\left(3.69 \times 10^{6}\right)$ & $28.77 \%\left(1.49 \times 10^{6}\right)$ & \multirow{2}{*}{0.488} \\
\cline { 1 - 3 } LLLT-SA & $70.56 \%\left(4.10 \times 10^{6}\right)$ & $29.44 \%\left(1.71 \times 10^{6}\right)$ & \multirow{2}{*}{0.472} \\
\cline { 1 - 3 } aPDT-B & $53.86 \%\left(2.79 \times 10^{6}\right)$ & $46.14 \%\left(2.39 \times 10^{6}\right)$ & \\
\hline aPDT-SA & $55.59 \%\left(3.23 \times 10^{6}\right)$ & $44.41 \%\left(2.58 \times 10^{6}\right)$ & \\
\hline
\end{tabular}

Abbreviations: aPDT, antimicrobial photodynamic therapy; B, periodontal biofilm; CFU, colony-forming unit; LLLT, low-level laser therapy; SA, Staphylococcus aureus.

*Statistically significant for $p<0.05$ (comparison of percentages).

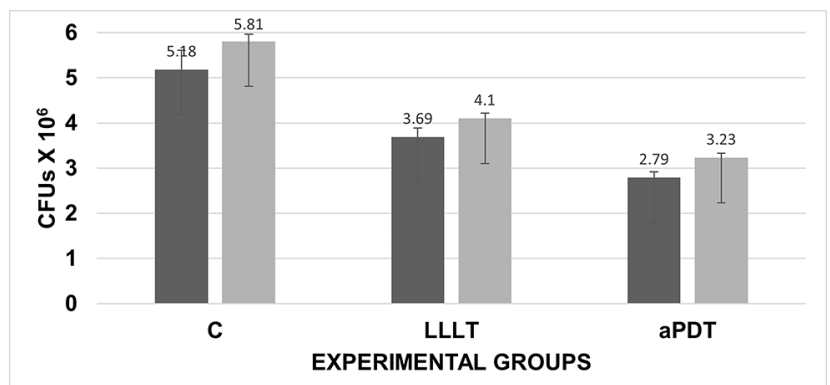

Fig. 3 Number of colony-forming units (CFUs) on the experimental groups. Control (C), low-level laser therapy (LLLT), and antimicrobial photodynamic therapy (aPDT). Periodontal biofilm (dark bars); Staphylococcus aureus (light bars). Error bars indicate standard deviation (one-way ANOVA and post hoc Tukey's test). ANOVA, analysis of variance.

\section{Discussion}

The present study tested the LLLT and aPDT against periodontal biofilm and S. aureus in vitro, and the results showed good efficacy of both protocols.

As previously stated, peri-implantitis is a complex clinical condition that presents a variable and heterogeneous microbiota. ${ }^{6}$ From this perspective, finding which method is most effective against the microorganisms involved in the clinical condition of peri-implantitis is an important step in establishing protocols for treating the disease.

The literature shows that in vitro models of biofilm containing several species can serve as useful tools in studying various polymicrobial infections. ${ }^{25}$ Thus, this study used periodontal biofilm previously collected from a volunteer with periodontitis. ${ }^{20}$ This subgingival biofilm sample was selected due to the similarities with the microbiota presented in cases of peri-implantitis. ${ }^{9-11}$ The morphotinturial analysis, performed immediately after collection and before freezing, revealed the presence of gram-positive and gram-negative microorganisms $\mathrm{s}^{20}$; however, there was no characterization of this biofilm, with the identification of microorganisms, due to the need for more complex analyzes that demand higher costs and specific equipment.

The selected S. aureus strain is standard (ATCC 25923). S. aureus was chosen because it requires special attention throughout the peri-implantitis process due to its ability to firmly adhere to the titanium surface ${ }^{26}$ and its possible
Table 3 Means and standard deviations of the CFUs of the LLLT and aPDT groups compared separately according to microorganisms' culture

\begin{tabular}{|l|l|l|}
\hline Groups & CFUs & $p^{*}$ \\
\hline LLLT-B & $3.69 \times 10^{6}(0.20)$ & $0.000^{*}$ \\
\hline aPDT-B & $2.79 \times 10^{6}(0.13)$ & \\
\hline LLLT-SA & $4.10 \times 10^{6}(0.12)$ & $0.000^{*}$ \\
\hline aPDT-SA & $3.23 \times 10^{6}(0.10)$ & \\
\hline
\end{tabular}

Abbreviations: aPDT, antimicrobial photodynamic therapy; B, periodontal biofilm; CFU, colony-forming unit; LLLT, low-level laser therapy; SA, Staphylococcus aureus.

${ }^{*}$ Statistically significant for $p<0.05$ (independent $t$-test).

participation on the biofilm formation as a primary colonizer, creating favorable conditions for the adhesion of bacteria of late colonization. ${ }^{10}$

In this study, two protocols of antimicrobial effect using LLLT and aPDT were analyzed. The LLLT protocol was adapted from Salmeron et $\mathrm{al}^{19}$ for decontamination of titanium discs contaminated by oral biofilm in situ and evaluated in vivo. The adaptation was related to the fluency parameters and exposure time changed due to the equipment used that had these parameters prefixed according to protocol selection. The equipment was the same used by this research group ${ }^{20}$ with this irradiation protocol for aPDT. In the previous study, ${ }^{19}$ the fluency and exposure time were lower, $45 \mathrm{~J} / \mathrm{cm}^{2}$ and 30 seconds, respectively, and similar results were found, with good potential for decontamination of the discs using the LLLT.

The present results also suggest good efficacy of the LLLT in the parameters used, with a significant reduction in the number of CFUs in the periodontal biofilm and S. aureus groups compared with control groups. De Sousa et $\mathrm{al}^{27}$, used the same wavelength and power $(660 \mathrm{~nm} ; 30 \mathrm{~mW})$ and also obtained a reduction in the growth of $S$. aureus with the difference that they used lower fluences than used in this study.

Different effects can be seen in the action of the laser on gram-positive and gram-negative bacteria, ${ }^{27}$ probably due to structural differences that can affect the penetration of laser irradiation and mediate differences in susceptibility. ${ }^{27}$ It can be related and explain partially the similar results in reduction of CFUs presented in this study for periodontal biofilm and $S$. aureus, since there was gram-positive microorganisms in periodontal biofilm too. 
The studies still present controversial data regarding the antimicrobial action of the laser, considering the difficulty in standardizing the protocols due to the number of parameters and the type of laser used. For this reason, in the last decades, the association of LLLT with photosensitizers has also been used to reduce or eliminate bacteria, in the called aPDT. ${ }^{19,20,28}$ In this study, the aPDT protocol was the same used by Batalha et $\mathrm{a}^{20}$ and similar results were obtained. These authors used the same sample of periodontal biofilm to contaminate dental implants in vitro and have a significant reduction in the number of CFUs compared with control group.

The effects of aPDT involve several parameters, not only referring to the laser but the dye as well. The interaction of these photosensitizer dyes with the laser wavelength is essential for the correct functioning of the mechanism of action of aPDT. ${ }^{29}$ Researchers have preferred TBO for interacting better with lipopolysaccharides from gram-negative bacteria even without a light source, ${ }^{30}$ according to some studies reporting the use of the dye alone. ${ }^{19,28}$ Besides, ТBO has an intense absorption in the region of 620 to $660 \mathrm{~nm}^{29}$ which justifies selecting the wavelength used in this study. The preirradiation time of 1 minute was also defined based on literature. ${ }^{19,20,28}$

Although the reduction in the number of CFUs for both cultures with both protocols was quite satisfactory, it has not yet been sufficient to eliminate microorganisms. This indicates that the association of physical and chemical methods, as suggested by Cai et $a l,{ }^{16}$ can be used for decontamination of dental implant surfaces. Another interesting finding was the significant difference between the aPDT results compared with LLLT results, separately for both cultures. The aPDT reduced the number of CFUs more than the LLLT for periodontal biofilm and S. aureus, with better antimicrobial action. And the efficacy of both treatments was similar against periodontal biofilm and $S$. aureus.

\section{Limitations}

It is noted that the use of LLLT and aPDT is effective in combating the growth of several microorganisms, especially the aPDT that presented a superior antimicrobial action, suggesting that it can be an adjunct therapy option in the treatment of peri-implant diseases. However, protocols and therapies that employ the laser have limitations, that is, there are no specific forms of application, emphasizing the need for further studies to improve the preexisting protocols, since these therapies are very promising.

\section{Conclusion}

Based on the results obtained and considering the limitations of this in vitro study, it was possible to conclude that the protocols of LLLT and aPDT used were effective in reducing cultures of periodontal biofilm and S. aureus, with the superiority of antimicrobial photodynamic therapy.

\section{Conflict of Interest}

None declared.

\section{Acknowledgment}

Authors thank Vanessa C. Batalha to provide the laser equipment used in this study.

\section{References}

1 Quirynen M, Herrera D, Teughels W, Sanz M. Implant therapy: 40 years of experience. Periodontol 2014;66(1):7-12

2 Pjetursson BE, Asgeirsson AG, Zwahlen M, Sailer I. Improvements in implant dentistry over the last decade: comparison of survival and complication rates in older and newer publications. Int J Oral Maxillofac Implants 2014;29(Suppl): 308-324

3 Sinjab K, Garaicoa-Pazmino C, Wang HL. Decision making for management of periimplant diseases. Implant Dent 2018;27(3):276-281

4 Caton JG, Armitage G, Berglundh T, et al. A new classification scheme for periodontal and peri-implant diseases and conditions - Introduction and key changes from the 1999 classification. J Periodontol 2018;89(Suppl 1):S1-S8

5 Heitz-Mayfield LJA, Salvi GE. Peri-implant mucositis. J Periodontol 2018;89(Suppl 1):S257-S266

6 Schwarz F, Derks J, Monje A, Wang HL. Peri-implantitis. J Periodontol 2018;89(Suppl 1):S267-S290

7 Berglundh T, Armitage G, Araujo MG, et al. Peri-implant diseases and conditions: consensus report of workgroup 4 of the 2017 World Workshop on the Classification of Periodontal and Peri-Implant Diseases and Conditions. J Clin Periodontol 2018;45(Suppl 20):S286-S291

8 Lang NP, Brägger U, Walther D, Beamer B, Kornman KS. Ligature-induced peri-implant infection in cynomolgus monkeys. I. Clinical and radiographic findings. Clin Oral Implants Res 1993;4(1):2-11

9 Pérez-Chaparro PJ, Duarte PM, Shibli JA, et al. The current weight of evidence of the microbiologic profile associated with peri-implantitis: a systematic review. J Periodontol 2016;87(11):1295-1304

10 Persson GR, Renvert S. Cluster of bacteria associated with peri-implantitis. Clin Implant Dent Relat Res 2014;16(6):783-793

11 RenvertS, Quirynen M.Riskindicators for peri-implantitis.Anarrative review. Clin Oral Implants Res 2015;26(suppl 11):15-44

12 Klinge B, Klinge A, Bertl K, Stavropoulos A. Peri-implant diseases. Eur J Oral Sci 2018;126(Suppl 1):88-94

13 Padial-Molina M, López-Martínez J, O’Valle F, Galindo-Moreno P. Microbial profiles and detection techniques in peri-implant diseases: a systematic review. J Oral Maxillofac Res 2016;7(3):e10

14 Mombelli A, Décaillet F. The characteristics of biofilms in peri-implant disease. J Clin Periodontol 2011;38(Suppl 11): 203-213

15 Sahrmann P, Gilli F, Wiedemeier DB, Attin T, Schmidlin PR, Karygianni L. The microbiome of peri-implantitis: a systematic review and meta-analysis. Microorganisms 2020;8(5):8

16 Cai Z, Li Y, Wang Y, et al. Antimicrobial effects of photodynamic therapy with antiseptics on Staphylococcus aureus biofilm on titanium surface. Photodiagn Photodyn Ther 2019;25:382-388

17 Heitz-Mayfield LJ, Lang NP. Comparative biology of chronic and aggressive periodontitis vs. peri-implantitis. Periodontol 2000 2010;53:167-181

18 Fraga RS, Antunes LAA, Fontes KBFDC, Küchler EC, Iorio NLPP, Antunes LS. Is antimicrobial photodynamic therapy effective for microbial load reduction in peri-implantitis 
treatment? A systematic review and meta-analysis. Photochem Photobiol 2018;94(4):752-759

19 Salmeron S, Rezende ML, Consolaro A, et al. Laser therapy as an effective method for implant surface decontamination: a histomorphometric study in rats. J Periodontol 2013;84(5):641-649

20 Batalha VC, Bueno RA, Fronchetti Junior E, et al. Dental implants surface in vitro decontamination protocols. Eur J Dent 2021;15(3):407-411 doi:10.1055/s-0040-1721550

21 Lerario F, Roncati M, Gariffo A, et al. Non-surgical periodontal treatment of peri-implant diseases with the adjunctive use of diode laser: preliminary clinical study. Lasers Med Sci 2016;31(1):1-6

22 Deppe H, Horch HH, Henke J, Donath K. Peri-implant care of ailing implants with the carbon dioxide laser. Int J Oral Maxillofac Implants 2001;16(5):659-667

23 Mailoa J, Lin GH, Chan HL, MacEachern M, Wang HL. Clinical outcomes of using lasers for peri-implantitis surface detoxification: a systematic review and meta-analysis. J Periodontol 2014;85(9):1194-1202

24 Lin GH, Suárez López Del Amo F, Wang HL. Laser therapy for treatment of peri-implant mucositis and peri-implantitis: an American Academy of Periodontology best evidence review. J Periodontol 2018;89(7):766-782
25 Thurnheer T, Belibasakis GN. Incorporation of staphylococci into titanium-grown biofilms: an in vitro "submucosal" biofilm model for peri-implantitis. Clin Oral Implants Res 2016;27(7):890-895

26 Harris LG, Richards RG. Staphylococcus aureus adhesion to different treated titanium surfaces. J Mater Sci Mater Med 2004;15(4):311-314

27 de Sousa NTA, Gomes RC, Santos MF, Brandino HE, Martinez R, de Jesus Guirro RR. Red and infrared laser therapy inhibits in vitro growth of major bacterial species that commonly colonize skin ulcers. Lasers Med Sci 2016;31(3):549-556

28 Valle LA, Lopes MM, Zangrando MSR, et al. Blue photosensitizers for aPDT eliminate Aggregatibacter actinomycetemcomitans in the absence of light: An in vitro study. J Photochem Photobiol B 2019;194:56-60

29 Passanezi E, Damante CA, de Rezende ML, Greghi SL. Lasers in periodontal therapy. Periodontol 2000 2015;67(1):268-291

30 Usacheva MN, Teichert MC, Biel MA. Comparison of the methylene blue and toluidine blue photobactericidal efficacy against gram-positive and gram-negative microorganisms. Lasers Surg Med 2001;29(2):165-173 